\section{Agios' high metabolism}

\section{By Steven Edelson, Executive Editor}

Massachusetts Institute of Technology, Agios Pharmaceuticals Inc. and other collaborators have used an RNAi-based in vivo screening technology to discover cancer targets directly related to tumor metabolism. The team applied the approach to a subset of metabolic genes and identified phosphoglycerate dehydrogenase, a key enzyme in serine biosynthesis, as being involved in breast cancer. ${ }^{1}$

The same team is now screening the complete set of human metabolic genes to identify additional cancer targets.

"The whole premise of starting Agios was looking for new oncology targets and coming at cancer from a different angle than signal transduction and signaling networks-instead looking at metabolic enzymes rewired by cancers to feed their needs and growth potential,"
"The published work is basically a pilot screen now we're screening the entire metabolome."

-Scott Biller,

Agios Pharmaceuticals Inc.
Next, the group used an RNAi-based approach to screen the genes in mice. The list of resulting hits was then prioritized based on known cancer-related genomic amplification data, resulting in the identification of $\mathrm{PHGDH}$ as essential for tumorigenesis.

The team also used flux analysis to determine how the metabolic fluxes around the serine pathway were different in cells with high PHGDH expression compared with in cells that had normal expression. In breast cancer cell lines with high levels of PHGDH, inhibiting the target decreased cell proliferation.

Results were published in Nature.

Sabatini is on Agios' scientific advisory board (SAB). The team also included researchers from Harvard University.

Separately, a Harvard Medical School-MIT team came to similar conclusions about PHGDH's role in oncogenesis. That group used an NMR-based spectroscopy approach to study metabolic fluxes involved in diverting glucose-derived metabolites toward cancer cell proliferation. Those findings were published in Nature Genetics. ${ }^{3}$

This second team was led by Matthew Vander Heiden, assistant professor of biology at MIT, and Lewis Cantley, professor of systems biology at Harvard Medical School. Both are members of Agios' SAB.

"All cancer cells need to solve a problem: the conversion of glutamate to $\alpha$-ketoglutarate," an intermediate in the citric acid cycle that is used by cancer cells to create backbones necessary to synthesize both nucleotides and said CEO David Schenkein. "We said from the start that a core competency we needed to build was the ability to do flux biochemistry studies to see how metabolites are moving. This paper is a glimpse at one of the toolsets we've created."

Flux is the rate of metabolite turnover in a cell. Cancer cells require high metabolic fluxes of carbon metabolism-derived metabolites that are used as cellular building blocks to sustain tumor growth.

Whereas previous research had shown that estrogen receptornegative breast cancer patients had higher levels of phosphoglycerate dehydrogenase (PHGDH) mRNA than estrogen receptor-negative patients, ${ }^{2}$ the current study provides the first proof that this enzyme is metabolically linked to tumor proliferation.

PHGDH catalyzes the first step in the serine biosynthesis pathway. Some cancer cells hijack that pathway and use it to ramp up production of proteins and nucleotides required for proliferation.

The team, led by David Sabatini, a member of the Whitehead Institute for Biomedical Research and an associate professor of biology at MIT, started with a list of 2,752 genes that encode all known human metabolic enzymes and small molecule transporters. From there, the researchers used publicly available oncogenomic information to whittle down the list to 133 genes most likely to yield cancer targets.

For example, the list included genes differentially expressed in tumor versus normal tissues or genes already known to be highly expressed in aggressive breast cancer. nucleic acids, said lead author Richard Possemato, a postdoctoral fellow at the Whitehead Institute. "In the ER-negative breast cancer cells we used, the serine biosynthesis pathway provides a significant amount of that flux."

The citric acid cycle helps convert carbohydrates, fats and proteins into carbon dioxide and water to generate energy for the cell.

Agios CSO Scott Biller said the in vivo aspect of the screening technology "is very important-we've all been misled by things that happen in a dish. Knocking out genes in a multiplexed format in an actual tumor is a powerful technology."

Both Agios and the Whitehead Institute now want to expand the screening technology to the entire set of genes for human metabolic enzymes and transporters.

"The paper picked out the high-priority genes, but we want to have a broader systems approach," said Possemato. "We want to tease out any pathways and start to get a big-picture view of the essentiality of cancer metabolism."

Added Biller, "The published work is basically a pilot screennow we're screening the entire metabolome."

The screening is being done in collaboration with Sabatini.

As for PHGDH itself, Agios hasn't disclosed what its plans are. The company's lead program, which is in preclinical development,

\section{All encompassing}




\section{ANALYSIS}

\section{TARGETS \& MECHANISMS}

focuses on a cancer metabolism target called isocitrate dehydrogenase 1 (IDH1). Agios also has a discovery-stage program focused on a different cancer metabolism target-pyruvate kinase M2 isozyme (PKM2).

Edelson, S. SciBX 4(30); doi:10.1038/scibx.2011.842

Published online Aug. 4, 2011

REFERENCES

1. Possemato, R. et al. Nature; published online July 14, 2011; doi:10.1038/nature10350

Contact: David M. Sabatini, Whitehead Institute for Biomedical Research, Cambridge, Mass.

e-mail: sabatini@wi.mit.edu
2. Pollari, S. et al. Breast Cancer Res. Treat. 125, 421-430 (2011)

3. Locasale, J.W. et al. Nat. Genet.; published online July 31, 2011; doi:10.1038/ng.890

Contact: Matthew G. Vander Heiden, Massachusetts Institute of Technology, Cambridge, Mass.

e-mail: mvh@mit.edu

Contact: Lewis C. Cantley, Harvard Medical School, Boston, Mass. e-mail: Icantley@hms.harvard.edu

COMPANIES AND INSTITUTIONS MENTIONED

Agios Pharmaceuticals Inc., Cambridge, Mass.

Harvard Medical School, Boston, Mass.

Harvard University, Cambridge, Mass.

Massachusetts Institute of Technology, Cambridge, Mass.

Whitehead Institute for Biomedical Research, Cambridge, Mass. 